\title{
Potential of colors for determining age and metallicity of stellar populations
}

\author{
Z. $\mathrm{Li}^{1,2}$, Z. Han ${ }^{1}$, and F. Zhang ${ }^{1}$ \\ 1 National Astronomical Observatories/Yunnan Observatory, the Chinese Academy of Sciences, Kunming, 650011, PR China \\ e-mail: zhongmu.li@gmail.com \\ 2 Graduate School of the Chinese Academy of Sciences, PR China
}

Received 4 September 2006 / Accepted 4 December 2006

\section{ABSTRACT}

\begin{abstract}
Context. Colors are usually not used for constraining stellar populations because they are thought to have the well-known agemetallicity degeneracy, but some recent works show that colors can also be used. A simple stellar population synthesis model is widely used, but there is no analysis for its colors.

Aims. We try to find colors that can potentially be used to determine the age and metallicity of stellar populations by the standard model.

Methods. Principal component analysis and relative sensitive parameter techniques are used in this work.

Results. $U-K, U-H, U-J, B-K, B-H, U-I, B-J$, and $V-K$ are found to be more important for studying populations than others. Pairs of colors such as $B-K$ and $B-V$ are found to be able to disentangle the stellar age-metallicity degeneracy via the high-resolution model, while pairs such as $U-K$ and $R-I$ may be used instead when the low-resolution model is used. Furthermore, the $u-g$ and $r-i$ colors of the low-resolution model seem to have the same potential, but there are no such colors for the high-resolution one. Conclusions. Some colors have been shown to have the potential to determine the age and metallicity of stellar populations, but relative metallicity and age sensitivities of colors in different stellar population synthesis models are usually different. In addition, minor star formations will make star systems look younger and more metal rich than their dominating populations.
\end{abstract}

Key words. galaxies: stellar content - galaxies: formation - galaxies: elliptical and lenticular, cD

\section{Introduction}

Determination of ages and metallicities of various stellar populations has long been a very important subject in astronomy and astrophysics because it can help us to understand not only galaxies, but also cosmology. Many such works are done via the popular technique called stellar population synthesis. Many simple stellar population (SSP) synthesis models, e.g., Vazdekis (1999), Worthey (1994), Bruzual \& Charlot (2003, hereafter BC03), and Zhang et al. (2005), are used widely and have shown us many new results.

To study properties of stellar populations, most people choose to use spectra-like methods, e.g., absorption line indices, to determine age and metallicity as they have the ability to break the well-known age-metallicity degeneracy. But, in fact, we all hope to use colors for such work because they are easier to get than spectra and are independent of the distances of objects. A lot of work has been done in this way and recent such works are given by, e.g., Dorman et al. (2003) and James et al. (2006). The uncertainties of using synthetic integrated colors of SSPs to determine stellar ages are investigated by Yi (2003).

However, different stellar population synthesis models and colors are usually used by different authors and it seems difficult to do future work using the same method because other people do not have the same models. Therefore, it is valuable to study some widely used stellar population synthesis models. In this paper, we intend to analyze the $\mathrm{BC} 03$ standard model, with the aim of finding some colors that have the potential to break the degeneracy between stellar age and metallicity.
The organization of the paper is as follows. In Sect. 2, we briefly introduce the $\mathrm{BC} 03$ model and techniques used in this paper. In Sect. 3, we analyze UBVRIJHK and ugriz colors of the BC03 standard model. Finally, we give our conclusions in Sect. 4

\section{BC03 model and techniques}

The stellar population synthesis model of BC03 is a widely used model in stellar population synthesis. Its standard model takes the Chabrier (2003) initial mass function (IMF) and uses Padova 1994 library tracks to calculate integrated colors. The model supplies us with both $U B V R I J H K$-kind and ugriz colors that are compared to observations from the Sloan Digital Sky Survey (SDSS) and galactic clusters. Thus it is convenient to use the model for estimating stellar populations via colors.

To search for colors, which are important to determine ages and metallicities of populations, we utilize two methods, i.e., principal component analysis (PCA) and relative metallicity sensitivity (rms) techniques. PCA is a powerful technique for unveiling the correlation between variables in a data set and for determining the intrinsic dimensionality of a parameter space (Connolly \& Szalay 1999). It can help us to reduce the dimension of variables and give the important variables in the initial data set. Therefore it can be used to find indices that have the potential to reveal properties of stellar populations (see Kong \& Cheng 2003, for more detail). The algorithm works by building more compact and optimal linear combinations of data with 
respect to the mean square error criterion and calculating a new base with the minimum set of orthogonal axes, which are called principal components (PCs), and can describe the variance of the data on the whole. The most important PC is called the first principal component (PC1) and can usually express the main information of the original data. Thus the importance of each input variable for expressing the main information of the source data (now represented by PC1) can be estimated via the weights assigned to them, i.e., their correlations with PC1. In this work, we intend to find colors important to stellar age or metallicity via PCA. The results can, possibly, guide us to choose important colors to determine populations by multi-color methods and may also show us some colors that are markedly important to stellar age or metallicity, as with the spectral indices of Kong \& Cheng (2003).

As we will show, PCA of colors fails to show colors that are markedly important to stellar age or metallicity. We investigate the relative age and metallicity sensitivities of colors via the rms technique, which can quantify how a color is sensitive to age and metallicity of SSPs. One can refer to Worthey (1994), in which rmss for some colors and absorption line indices are quantified.

\section{PCA results and metallicity sensitivities of colors}

\subsection{Main results}

To find colors important to stellar age, we analyze the colors of SSPs with the same metallicity and check their correlations to $\mathrm{PC} 1$, as PC1 expressed more than $94 \%$ of the information from the original data. Because the differences in colors result purely from stellar age and are expressed mainly by PC1, colors that correlate strongly to PC1 will be important to stellar age. A similar method is also used to look for colors important to stellar metallicity. Because UBVRIJHK and ugriz colors can usually be derived from many sources (e.g., SDSS and TwoMicron All-Sky Survey (2MASS)) and seem to be more available than others, we analyze them in this work. The main results for $U B V R I J H K$ colors of high-resolution SSPs are shown in Tables 1 and 2. In each table, the contribution of PC1 to the total variance and the weight assigned to each input color are shown. As the results for colors of low-resolution SSPs are very similar to those of the high-resolution ones, we do not list them here. We do not show those for ugriz colors either, as their PCA results are similar.

Tables 1 and 2 show the contribution of each PC1 for expressing the original information and correlations of colors to PC1, with a mainly descending order. Colors with big correlations are more important to age and metallicity than those with small correlations. In detail, $U-K, U-H, U-J, B-K, B-H$, $U-I, B-J$, and $V-K$ colors are shown to be more important than others to determine the ages and metallicities of stellar populations. In addition, most colors that are important to stellar metallicity are important to stellar age. Thus it is difficult to determine stellar age or metallicity via only a color. In other words, it is more appropriate to determine stellar age and metallicity of a population via a few colors. This result is in agreement with that of Chang et al. (2006) that optical colors of elliptical galaxies are sensitive to a combination of age, metallicity, and $\alpha$-enhancement.

As we want to find pairs of colors that are able to disentangle the age-metallicity degeneracy, we investigate the relative age and metallicity sensitivities of colors using a method similar to Worthey (1994). A rms is defined for each color to estimate how it is sensitive to age and metallicity. In detail, rms is the
Table 1. PCA results of UBVRIJHK colors of SSPs in the highresolution BC03 model. Each row except the first shows the results for colors of SSPs with the same metallicity. "Percent" and "Correlations" are the contribution of $\mathrm{PC} 1$ to the total variance and correlations of colors to PC1.

\begin{tabular}{lcccc}
\hline \hline$Z$ & 0.0001 & 0.0040 & 0.0200 & 0.0500 \\
\hline Percent & 99.72 & PC1 1 & PC1 & PC1 \\
\hline & \multicolumn{5}{c}{ Correlations } & 97.54 & 94.40 \\
$U-K$ & -0.376 & -0.378 & -0.379 & -0.379 \\
$U-H$ & -0.363 & -0.364 & -0.365 & -0.364 \\
$U-J$ & -0.320 & -0.321 & -0.323 & -0.326 \\
$B-K$ & -0.287 & -0.287 & -0.285 & -0.284 \\
$B-H$ & -0.274 & -0.273 & -0.271 & -0.269 \\
$U-I$ & -0.244 & -0.248 & -0.243 & -0.244 \\
$B-J$ & -0.231 & -0.230 & -0.229 & -0.230 \\
$V-K$ & -0.217 & -0.214 & -0.214 & -0.215 \\
$U-R$ & -0.202 & -0.207 & -0.204 & -0.205 \\
$V-H$ & -0.204 & -0.200 & -0.200 & -0.199 \\
$R-K$ & -0.174 & -0.171 & -0.175 & -0.174 \\
$U-V$ & -0.159 & -0.164 & -0.165 & -0.164 \\
$R-H$ & -0.161 & -0.157 & -0.161 & -0.159 \\
$V-J$ & -0.161 & -0.157 & -0.158 & -0.161 \\
$B-I$ & -0.155 & -0.157 & -0.148 & -0.148 \\
$I-K$ & -0.132 & -0.130 & -0.136 & -0.135 \\
$I-H$ & -0.119 & -0.116 & -0.122 & -0.120 \\
$R-J$ & -0.118 & -0.114 & -0.119 & -0.121 \\
$B-R$ & -0.113 & -0.116 & -0.110 & -0.109 \\
$U-B$ & -0.089 & -0.091 & -0.095 & -0.095 \\
$V-I$ & -0.086 & -0.084 & -0.078 & -0.079 \\
$I-J$ & -0.076 & -0.073 & -0.081 & -0.082 \\
$B-V$ & -0.070 & -0.073 & -0.071 & -0.069 \\
$J-K$ & -0.056 & -0.057 & -0.056 & -0.053 \\
$J-H$ & -0.043 & -0.043 & -0.042 & -0.038 \\
$V-R$ & -0.043 & -0.043 & -0.039 & -0.040 \\
$R-I$ & -0.042 & -0.041 & -0.038 & -0.039 \\
$H-K$ & -0.013 & -0.014 & -0.014 & -0.015 \\
\hline & & & & \\
\hline
\end{tabular}

ratio of percentage change of age to that of metallicity when they lead to the same change in a color, respectively. According to the definition, colors with large rmss $(>1.0)$ are more sensitive to metallicity and those with small rmss $(<1.0)$ are more sensitive to stellar age. In this work, we do not fix the zero point (see Worthey 1994, for comparison). The main results are shown in Table 3, in which the average rmss of UBVRIJHK and ugriz colors are listed. The first and third columns show the results for colors of the high-resolution BC03 model and the second and fourth for the low-resolution model.

The results suggest that rmss of colors in high- and lowresolution models are different, as we see in Table 3 . In the highresolution model $B-K$ and $B-V$, but for the low-resolution model it is $U-K$ and $R-I$ that are sensitive to stellar metallicity and age, respectively. Moreover, $u-g$ and $r-i$ colors of the low-resolution model are found to be sensitive to stellar metallicity and age, respectively, but they show different sensitivities in the high-resolution model. As a whole, our result agrees well with previous works that the age-metallicity degeneracy can be disentangled by using optical and infrared colors together. One can refer to previous work of James et al. (2006), in which $J-K$ and $B-K$ colors are used as the metallicity and age sensitive colors and the work of Peletier et al. (1990), in which $U-V$, $B-V$, and $V-K$ are used to constrain the stellar populations of 12 galaxies. 
Table 2. Similar to Table 1, but each row represents PCA result for colors of SSPs with the same age.

\begin{tabular}{lcccc}
\hline \hline$t / \mathrm{Gyr}$ & 2 & 8 & 14 & 20 \\
\hline Percent & 99.73 & 99.86 & 99.84 & 99.81 \\
\hline & \multicolumn{5}{c}{ Correlations } \\
$U-K$ & -0.370 & -0.384 & -0.387 & -0.389 \\
$U-H$ & -0.343 & -0.361 & -0.366 & -0.370 \\
$U-J$ & -0.307 & -0.326 & -0.334 & -0.341 \\
$B-K$ & -0.305 & -0.283 & -0.277 & -0.269 \\
$B-H$ & -0.278 & -0.261 & -0.256 & -0.251 \\
$U-I$ & -0.197 & -0.226 & -0.240 & -0.248 \\
$B-J$ & -0.242 & -0.226 & -0.224 & -0.221 \\
$V-K$ & -0.237 & -0.222 & -0.213 & -0.206 \\
$U-R$ & -0.165 & -0.193 & -0.206 & -0.214 \\
$V-H$ & -0.210 & -0.200 & -0.192 & -0.187 \\
$R-K$ & -0.206 & -0.191 & -0.181 & -0.175 \\
$U-V$ & -0.133 & -0.162 & -0.174 & -0.183 \\
$R-H$ & -0.178 & -0.168 & -0.160 & -0.156 \\
$V-J$ & -0.174 & -0.165 & -0.160 & -0.157 \\
$I-K$ & -0.174 & -0.158 & -0.147 & -0.141 \\
$R-J$ & -0.143 & -0.134 & -0.128 & -0.126 \\
$I-H$ & -0.147 & -0.136 & -0.126 & -0.123 \\
$B-I$ & -0.132 & -0.125 & -0.130 & -0.128 \\
$U-B$ & -0.065 & -0.100 & -0.110 & -0.120 \\
$I-J$ & -0.111 & -0.101 & -0.094 & -0.093 \\
$B-R$ & -0.100 & -0.093 & -0.096 & -0.095 \\
$V-I$ & -0.063 & -0.064 & -0.066 & -0.064 \\
$B-V$ & -0.068 & -0.062 & -0.064 & -0.064 \\
$J-K$ & -0.063 & -0.057 & -0.053 & -0.048 \\
$R-I$ & -0.032 & -0.033 & -0.034 & -0.033 \\
$J-H$ & -0.036 & -0.035 & -0.032 & -0.030 \\
$V-R$ & -0.032 & -0.031 & -0.032 & -0.031 \\
$H-K$ & -0.027 & -0.022 & -0.021 & -0.019 \\
\hline & & & &
\end{tabular}

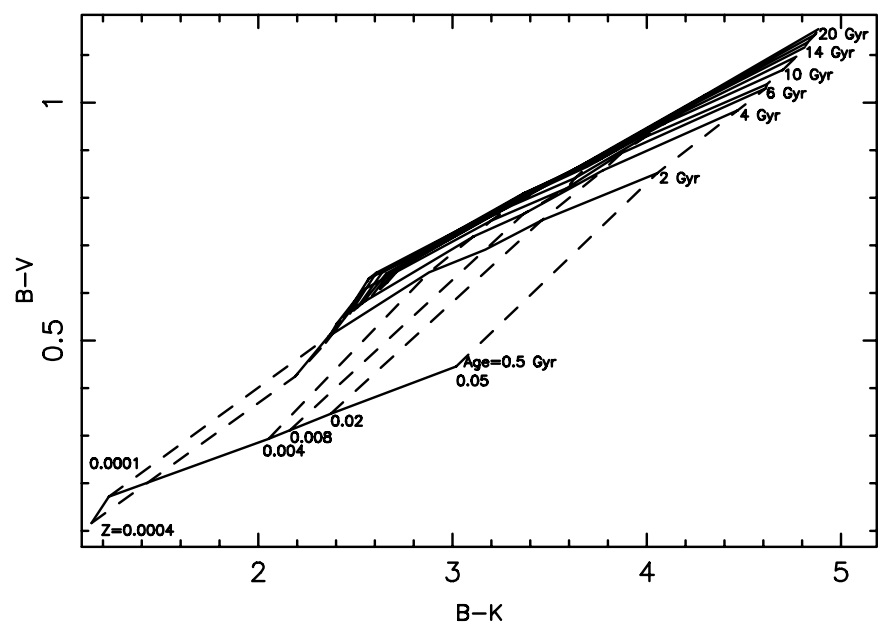

Fig. 1. $B-V$ vs. $B-K$ plane predicted by the high-resolution $\mathrm{BC} 03$ model. Solid and dashed lines represent constant age and constant metallicity, respectively.

\subsection{How colors can disentangle the age-metallicity degeneracy}

We plot some pairs of colors suggested by the above results to check their abilities for disentangling the stellar age-metallicity degeneracy and for constraining stellar populations, which can be seen in Figs. 1-3. The three figures plot pairs of $U B V R I J H K$ colors suggested for the high- and low-resolution models and ugriz colors for the low-resolution model, respectively. In Fig. 1,
Table 3. Relative metallicity sensitivities of colors. The left two columns show results for UBVRIHJK and the right two for ugriz colors. "High" and "Low" represent high and low resolution models of BC03, respectively.

\begin{tabular}{|c|c|c|c|c|c|c|c|}
\hline \multicolumn{2}{|r|}{ High } & \multicolumn{2}{|r|}{$\overline{\overline{\text { Low }}}$} & \multicolumn{2}{|r|}{$\overline{\text { High }}$} & \multicolumn{2}{|r|}{$\overline{\text { Low }}$} \\
\hline$B-K$ & 2.7029 & $U-K$ & 2.7077 & $u-z$ & 1.7542 & $u-g$ & 1.7598 \\
\hline$R-K$ & 2.2928 & $I-H$ & 2.3703 & $u-i$ & 1.5477 & $i-z$ & 1.4833 \\
\hline$I-H$ & 2.2870 & $R-J$ & 2.0011 & $r-z$ & 1.5225 & $u-i$ & 1.4609 \\
\hline$I-J$ & 2.0531 & $R-H$ & 1.9113 & $i-z$ & 1.4683 & $u-z$ & 1.4014 \\
\hline$U-K$ & 2.0250 & $R-K$ & 1.8018 & $g-i$ & 1.4370 & $u-r$ & 1.3825 \\
\hline$V-K$ & 1.9098 & $U-R$ & 1.7760 & $g-z$ & 1.3907 & $r-z$ & 1.3193 \\
\hline$V-H$ & 1.7760 & $I-J$ & 1.7533 & $g-r$ & 1.2722 & $g-z$ & 1.1709 \\
\hline$I-K$ & 1.7017 & $U-H$ & 1.7165 & $u-r$ & 1.2659 & $g-r$ & 1.0959 \\
\hline$U-H$ & 1.6686 & $V-H$ & 1.7116 & $r-i$ & 1.1425 & $g-i$ & 1.0437 \\
\hline$V-J$ & 1.6544 & $I-K$ & 1.6924 & $u-g$ & 1.0605 & $r-i$ & 0.7936 \\
\hline$R-J$ & 1.6201 & $V-K$ & 1.6696 & & & & \\
\hline$U-J$ & 1.5372 & $B-J$ & 1.6425 & & & & \\
\hline$R-H$ & 1.4821 & $U-J$ & 1.6153 & & & & \\
\hline$U-I$ & 1.3298 & $B-K$ & 1.5934 & & & & \\
\hline$B-I$ & 1.2154 & $U-I$ & 1.5452 & & & & \\
\hline$B-J$ & 1.1785 & $B-H$ & 1.5184 & & & & \\
\hline$B-H$ & 1.1669 & $U-V$ & 1.3781 & & & & \\
\hline$U-B$ & 0.9862 & $B-I$ & 1.3367 & & & & \\
\hline$B-R$ & 0.9483 & $V-J$ & 1.2083 & & & & \\
\hline$V-R$ & 0.7973 & $B-V$ & 1.0092 & & & & \\
\hline$U-V$ & 0.7362 & $B-R$ & 0.9572 & & & & \\
\hline$J-H$ & 0.7339 & $J-H$ & 0.9439 & & & & \\
\hline$V-I$ & 0.7132 & $V-I$ & 0.7675 & & & & \\
\hline$J-K$ & 0.6677 & $U-B$ & 0.7254 & & & & \\
\hline$H-K$ & 0.5506 & $V-R$ & 0.7137 & & & & \\
\hline$R-I$ & 0.4522 & $H-K$ & 0.5506 & & & & \\
\hline$U-R$ & 0.3974 & $J-K$ & 0.5290 & & & & \\
\hline$B-V$ & 0.3695 & $R-I$ & 0.4417 & & & & \\
\hline
\end{tabular}

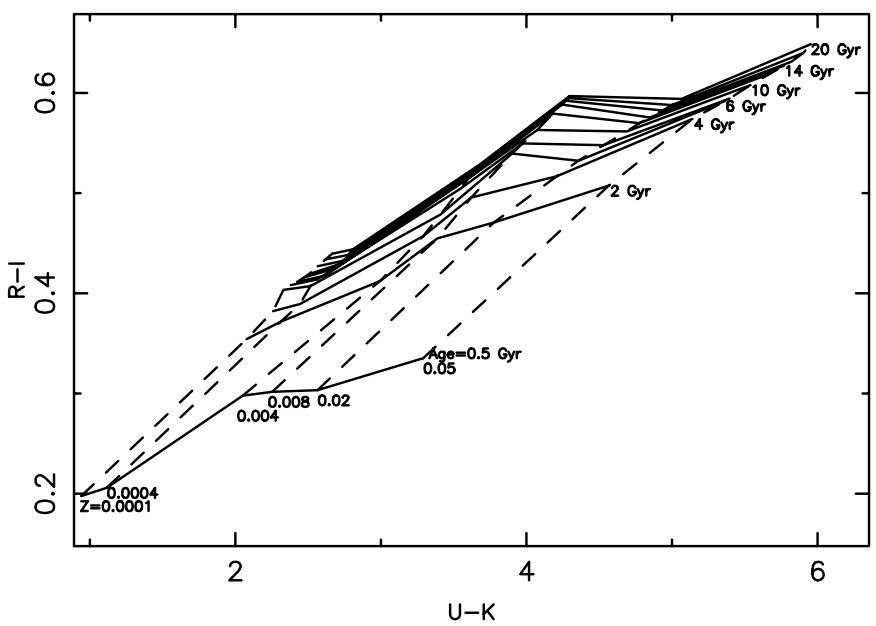

Fig. 2. $R-I$ vs. $U-K$ plane predicted by the low-resolution $\mathrm{BC} 03$ model. Lines have the same meanings as in Fig. 1.

$B-V$ and $B-K$, which are most likely to break the agemetallicity degeneracy via colors of the high-resolution BC03 model, are plotted. Constant age (isochrones) and constant metallicity are represented differently. It seems that the two colors have some potential to distinguish stellar populations. In Figs. 2 and 3, the similar relations for colors of the lowresolution $\mathrm{BC} 03$ model, i.e., $R-I$ versus $U-K$ and $r-i$ versus $u-g$ are plotted. We noticed that the AB-system colors suggested for the high-resolution model seem unable to separate the effects of age and metallicity and are not shown here. 


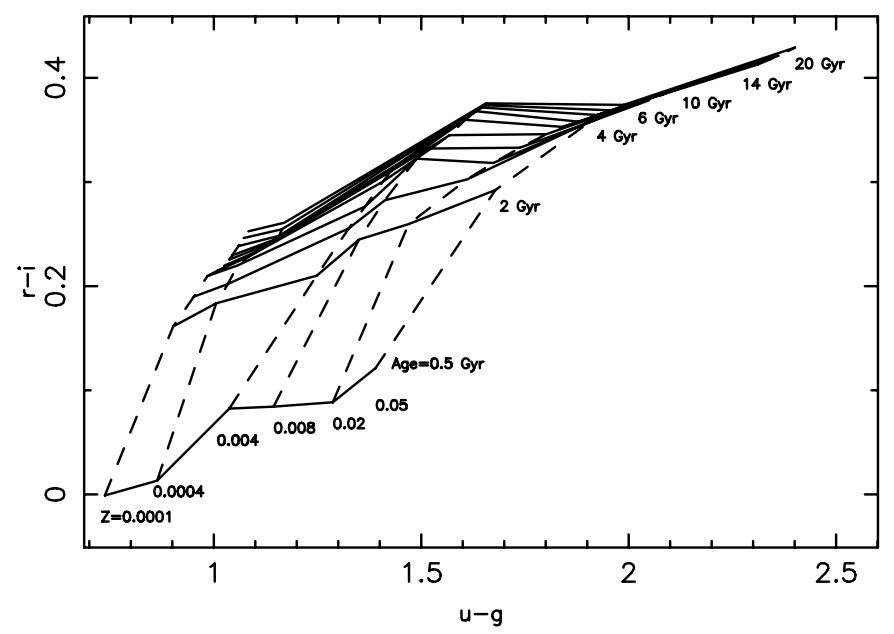

Fig. 3. $r-i$ vs. $u-g$ plane predicted by the low-resolution BC03 model. Lines have the same meanings as in Fig. 1.

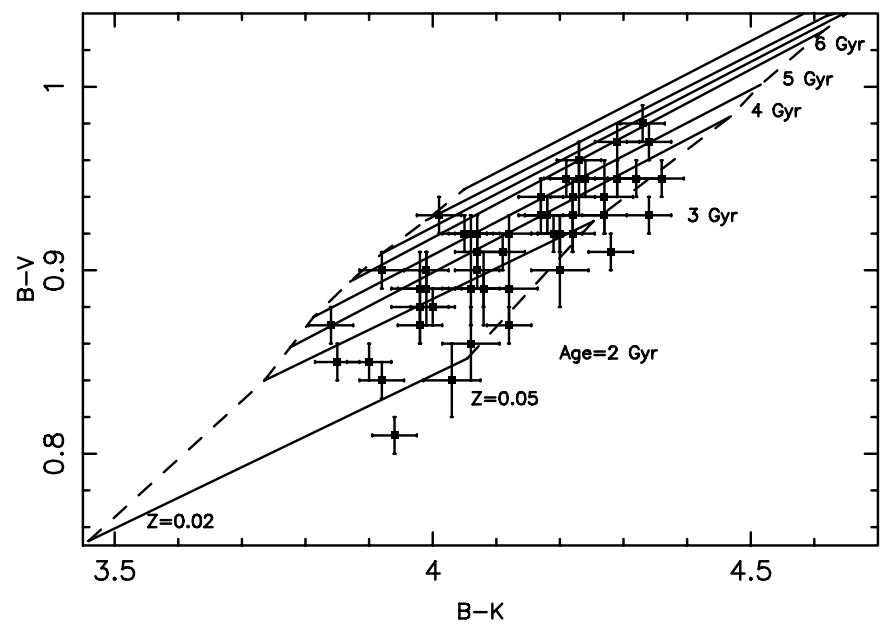

Fig. 4. Colors of 53 elliptical galaxies overlaid onto the $B-V$ vs. $B-K$ plane. Error bars show the uncertainties. Solid and dashed lines have the same meanings as in Fig. 1.

To compare with observation, we plot some observed galaxies in the $B-V$ versus $B-K$ plane and $r-i$ versus $u-g$ plane, which can be seen in Figs. 4 and 5. The first sample is selected from Michard (2005), consisting of 53 elliptical galaxies that have correct $U B V R I J H K$ photometry supplied and the second one from the Sloan Digital Sky Survery Data Release Five (SDSS-DR5), consisting of 12901 galaxies with concentration index $C \geq 2.8$, absolute $r$-band Petrosian magnitude $M_{r} \leq-22$, redshift $0.1 \leq z<0.25, r$-band Petrosian magnitude $14.5<r<17.77$, and with the best quality in "Photoz". The $k$-correction, galactic extinction, and differences between SDSS ugriz magnitudes and the AB-system are taken into account using the data supplied by SDSS, in a cosmology with $\Omega=0.3$, $\Lambda=0.7$, and $H_{0}=70 \mathrm{~km} \mathrm{~s}^{-1} \mathrm{Mpc}^{-1}$. We see that some galaxies are out of the grids, which perhaps results from the limit of the theoretical model and ongoing star formations. The detailed parameters of galaxies in the first sample are shown in Table 4, in which best-fitted metallicities, ages, and their associated $1 \sigma$ uncertainties of galaxies are listed. The results show that stellar ages and metallicities of these galaxies can be disentangled using the BC03 model, with average relative uncertainties of 0.26 and 0.13 in age and metallicity, respectively. However, most of these galaxies are shown to be very young and richer than solar

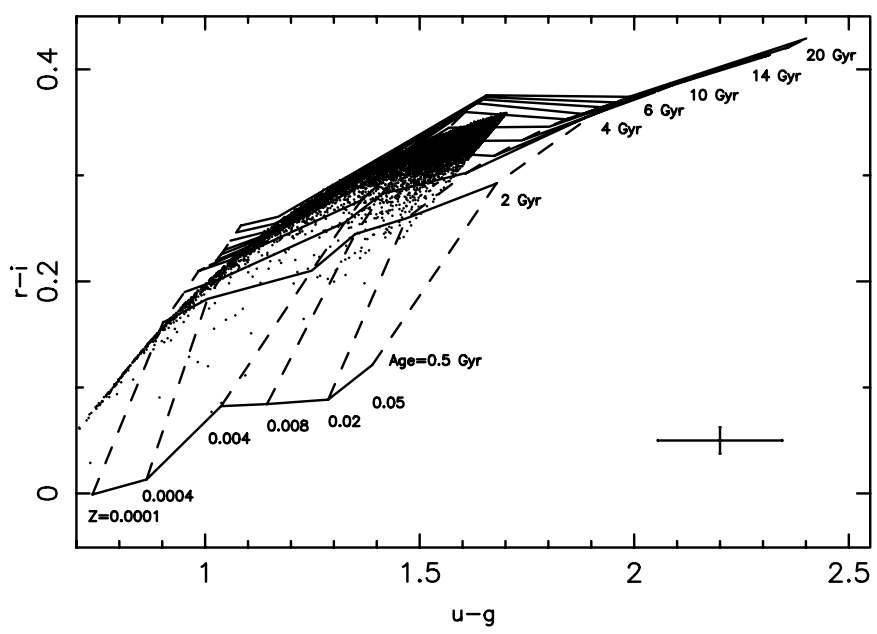

Fig. 5. $r-i$ and $u-g$ colors of 12901 early-type galaxies overlaid onto the theoretical calibration. Error bars show the typical uncertainties. Solid and dashed lines have the same meanings as in Fig. 1.

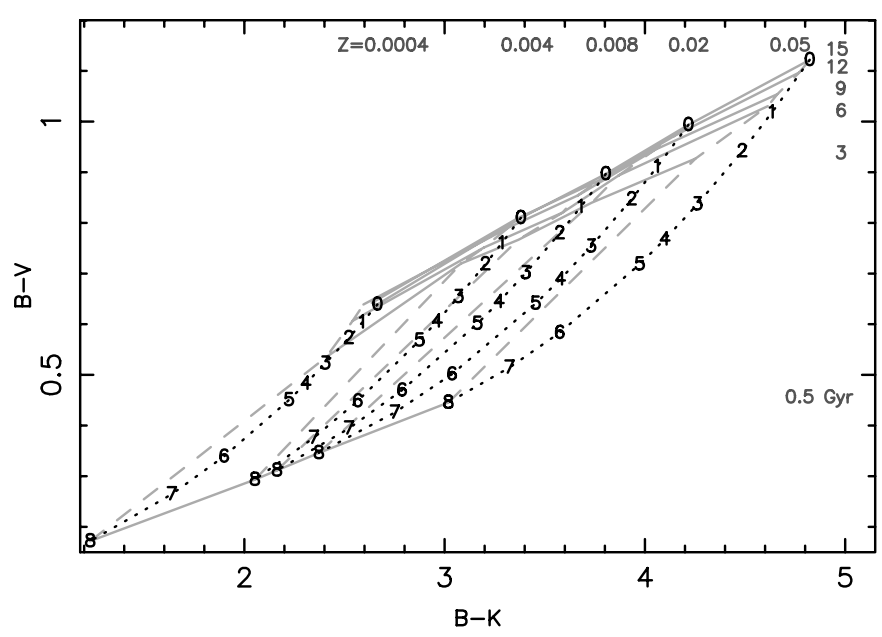

Fig. 6. Changes of $B-K$ and $B-V$ colors when mixing a young stellar population into an old one. The two stellar populations have the same metallicity. Solid and dashed lines are same as in Fig. 1. Dotted lines represent constant metallicity. Numbers from 0 to 8 show colors of an old population $(15 \mathrm{Gyr})$ mixed with a young population $(0.5 \mathrm{Gyr})$ with different percentages. From 0 to 8 , the young population contributes 0 , $0.5,1.0,2.0,3.0,4.0,10.0,20.0$, and 100 per cent in mass to the total system.

metallicity, which possibly results from minor star formations in them. In fact, recent star formations can change colors of a population significantly. Here we test the changes in Fig. 6 by mixing a young stellar population $(0.5 \mathrm{Gyr})$ into an old population (15 Gyr) with different percentages. Two stellar populations have the same metallicity. The numbers from 0 to 8 in the figure show that the young population contributes $0,0.5,1.0,2.0,3.0$, $4.0,10.0,20.0$, and 100 per cent in mass to the total system. As seen in Fig. 6, a young stellar population, even if it contributes to the whole system by only 0.5 per cent, will change the colors of the star system significantly, which makes the system look more metal rich and younger. This result is similar to that of line-strength indices of such systems (see, e.g., Serra \& Trager 2007). Therefore, the presence of galaxies that are out of the grid may result from recent star formations, and the dominating stellar populations of our sample galaxies, which contribute the most mass to these systems, are possibly older and less metal 
Table 4. Stellar ages and metallicities of 53 elliptical galaxies and the associated $1 \sigma$ uncertainties, fitted by $\mathrm{BC} 03$ using $B-K$ and $B-V$ colors.

\begin{tabular}{|c|c|c|c|c|}
\hline Name & $\begin{array}{c}\text { Age } \\
\text { [Gyr] }\end{array}$ & $\begin{array}{l}\text { Error } \\
{[\mathrm{Gyr}]}\end{array}$ & $\overline{Z Z}$ & Error \\
\hline NGC 0584 & 3.126 & 0.720 & 0.038 & 0.005 \\
\hline NGC 0596 & 4.416 & 1.066 & 0.029 & 0.003 \\
\hline NGC 0720 & 7.762 & 4.540 & 0.022 & 0.006 \\
\hline NGC 0821 & 5.370 & 1.086 & 0.035 & 0.003 \\
\hline NGC 1052 & 3.758 & 1.196 & 0.039 & 0.004 \\
\hline NGC 1395 & 3.802 & 1.153 & 0.043 & 0.005 \\
\hline NGC 1399 & 5.070 & 1.179 & 0.042 & 0.004 \\
\hline NGC 1404 & 5.821 & 2.031 & 0.037 & 0.004 \\
\hline NGC 1407 & 3.126 & 0.463 & 0.044 & 0.006 \\
\hline NGC 1537 & 2.786 & 0.845 & 0.044 & 0.010 \\
\hline NGC 1549 & 3.055 & 0.453 & 0.046 & 0.006 \\
\hline NGC 1600 & 3.388 & 1.130 & 0.048 & 0.005 \\
\hline NGC 1700 & 2.786 & 0.304 & 0.044 & 0.005 \\
\hline NGC 2768 & 3.350 & 2.021 & 0.031 & 0.009 \\
\hline NGC 2974 & 4.315 & 1.042 & 0.037 & 0.005 \\
\hline NGC 2986 & 3.311 & 0.149 & $\geq 0.05$ & \\
\hline NGC 3115 & 2.951 & 0.360 & 0.049 & 0.005 \\
\hline NGC 3193 & 5.070 & 1.025 & 0.030 & 0.003 \\
\hline NGC 3250 & 2.818 & 0.237 & $\geq 0.05$ & \\
\hline NGC 3377 & 4.074 & 0.996 & 0.023 & .003 \\
\hline NGC 3379 & 3.055 & 0.295 & $\geq 0.05$ & \\
\hline NGC 3557 & 2.570 & 0.628 & 0.048 & 0.008 \\
\hline NGC 3608 & 5.248 & 1.283 & 0.029 & 0.003 \\
\hline NGC 3610 & 1.603 & 0.216 & 0.049 & 0.004 \\
\hline NGC 3962 & 3.311 & 1.877 & 0.044 & 0.007 \\
\hline NGC 4125 & 3.508 & 2.116 & 0.030 & 0.010 \\
\hline NGC 4261 & 6.026 & 2.010 & 0.035 & 0.004 \\
\hline NGC 4278 & 2.917 & 0.281 & 0.037 & 0.004 \\
\hline NGC 4365 & 3.758 & 1.196 & 0.039 & 0.004 \\
\hline NGC 4374 & 3.236 & 1.079 & 0.039 & 0.005 \\
\hline NGC 4406 & 4.898 & 1.052 & 0.031 & 0.004 \\
\hline NGC 4472 & 3.388 & 0.119 & $\geq 0.05$ & \\
\hline NGC 4473 & 2.754 & 0.271 & 0.039 & 0.005 \\
\hline NGC 4478 & 2.851 & 0.251 & 0.030 & 0.004 \\
\hline NGC 4486 & 3.090 & 0.139 & $\geq 0.05$ & \\
\hline NGC 4494 & 2.630 & 0.339 & 0.036 & 0.004 \\
\hline NGC 4552 & 5.129 & 0.967 & 0.035 & 0.003 \\
\hline NGC 4564 & 5.689 & 2.347 & 0.023 & 0.005 \\
\hline NGC 4589 & 2.113 & 0.487 & 0.049 & 0.004 \\
\hline NGC 4621 & 3.055 & 0.453 & 0.046 & 0.006 \\
\hline NGC 4636 & 2.884 & 1.333 & 0.041 & 0.009 \\
\hline NGC 4649 & 6.310 & 2.008 & 0.038 & 0.003 \\
\hline NGC 4660 & 3.802 & 1.153 & 0.043 & 0.005 \\
\hline NGC 4696 & 3.350 & 1.959 & 0.046 & 0.008 \\
\hline NGC 4697 & 2.985 & 1.585 & 0.035 & 0.006 \\
\hline NGC 5322 & 1.928 & 0.286 & $\geq 0.05$ & \\
\hline NGC 5576 & 2.188 & 0.295 & 0.040 & 0.004 \\
\hline NGC 5813 & 4.365 & 1.054 & 0.039 & 0.005 \\
\hline NGC 5846 & 5.070 & 2.965 & 0.037 & 0.008 \\
\hline NGC 5866 & 2.213 & 0.186 & $\geq 0.05$ & \\
\hline NGC 7144 & 3.846 & 1.052 & 0.033 & 0.004 \\
\hline NGC 7507 & 3.936 & 1.019 & 0.035 & 0.004 \\
\hline IC1 459 & 4.898 & 1.225 & 0.039 & 0.004 \\
\hline
\end{tabular}

rich than that shown in Table 4. Our work also shows that if the young stellar population is older than about $1 \mathrm{Gyr}$ and takes only a small percentage, the metallicities measured from $B-K$ and $B-V$ are similar to their dominating populations, while the derived ages bias to their younger populations (see Fig. 7 in more detail). Thus the metallicities of our sample galaxies are credible and can be used for future studies.

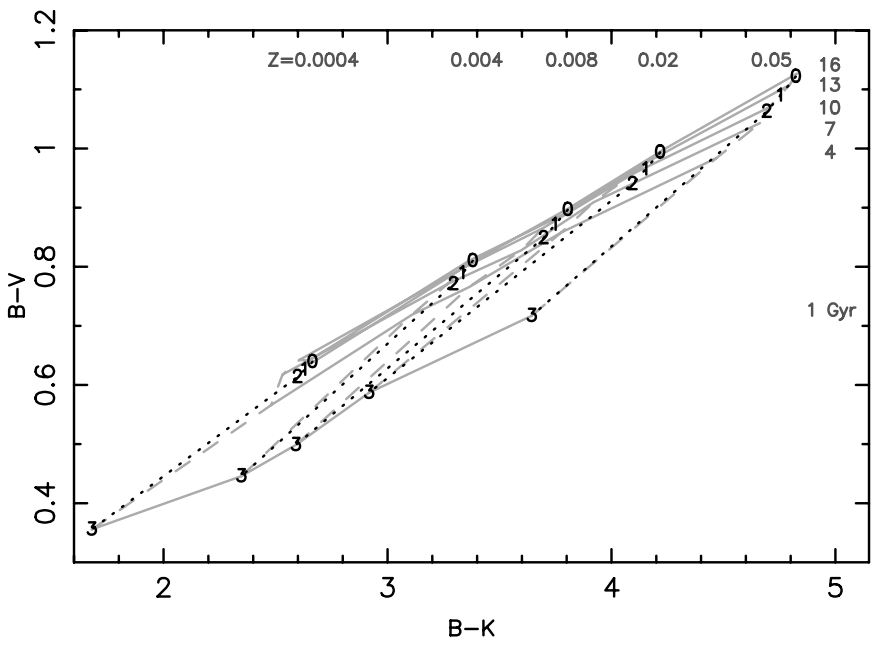

Fig. 7. Similar to Fig. 6, but for a composite system which includes a 15 Gyr stellar population and a 1 Gyr stellar population. Numbers from 0 to 3 mean that the young population contributes $0,0.5,1.0$, and 100 per cent in mass to the total system.

\section{Conclusions}

We analyzed $U B V R I J H K$ and ugriz colors of high- and lowresolution models of $\mathrm{BC} 03$ via $\mathrm{PCA}$ and rms techniques. The PCA show that colors such as $U-K, U-H, U-J, B-K$, $B-H, U-I, B-J$, and $V-K$ colors are better than others for determining stellar populations via multi-color methods. The rms results show that rmss of colors in different SSP models are very different from each other, even for the same model with just two different resolutions. For the high-resolution model of BC03, $B-K$ and $B-V$ are found to be sensitive, respectively, to stellar metallicity and age, and are possibly suitable for constraining populations. $U-K$ and $R-I$ should be used instead for the low-resolution model. In addition, $u-g$ and $r-i$ colors of the low-resolution model are shown to have the potential to constrain the stellar metallicity and age of populations. The results also show that it is better to use optical and infrared colors together for determining populations. However, if there are ongoing star formations, we will get smaller ages and richer metallicities than their dominating populations.

Acknowledgements. We gratefully acknowledge Dr. Richard Simon Pokorny for checking the English and Prof. Xu Kong for some useful discussions. We also thank the anonymous referee for useful comments that helped clarify some important points of the paper. This work is supported by the Chinese National Science Foundation (Grant Nos. 10433030, 10521001, and 10303006), the Chinese Academy of Sciences (No. KJX2-SW-T06), and Yunnan Natural Science Foundation (Grant No. 2005A0035Q).

\section{References}

Bruzual, G., \& Charlot, S. 2003, MNRAS, 344, 1000 Chabrier, G. 2003, ApJ, 586, L133

Chang, R. X., Gallazzi, A., Kauffmann, G., et al. 2006, MNRAS, 366, 717 Connolly, A. J., \& Szalay, A. S. 1999, AJ, 117, 2052

Dorman, B., O'Connell, R. W., \& Rood, R. T. 2003, ApJ, 591, 878

James, P. A., Salaris, M., Davies, J. I., et al. 2006, MNRAS, 367, 339

Kong, X., \& Cheng, F. Z. 2001, MNRAS, 323, 1035

Michard, R. 2005, A\&A, 441, 451

Peletier, R. F., Valentijin, E. A., \& Jameson, R. F. 1990, A\&A, 233, 62

Serra, P., \& Trager, S. C. 2007, MNRAS, 374, 769

Vazdekis, A. 1999, ApJ, 513, 224

Worthey, G. 1994, ApJS, 95, 107

Yi, S. 2003, ApJ, 582, 202

Zhang, F., Li, L., \& Han, Z. 2005, MNRAS, 364, 503 